\title{
Conversion between surface acoustic waves and guided modes of a quasi-periodic structured nanobeam
}

\author{
Alexander V. Korovin ${ }^{1}$, Yan Pennec ${ }^{1}$, Matteo Stocchi ${ }^{2}$, Davide Mencarelli, ${ }^{2,3}$, Luca \\ Pierantoni $^{2,3}$, Tapani Makkonen ${ }^{4}$, Jouni Ahopelto ${ }^{4}$ and Bahram Djafari Rouhani ${ }^{1}$ \\ ${ }^{1}$ IEMN, UMR CNRS 8520, University of Lille, 59652 Villeneuve d'Ascq, France \\ ${ }^{2}$ Department of Information Engineering, Marche Polytechnic University, Ancona, Italy \\ ${ }^{3}$ Laboratori Nazionali di Frascati (LNF), Istituto Nazionale di Fisica Nucleare (INFN), Frascati, Roma, \\ Italy \\ ${ }^{4}$ VTT Technical Research Centre of Finland Ltd, Finland \\ E-mail: xxx@xxx.xx
}

Received xxxxxx

Accepted for publication $\mathrm{xxxxxx}$

Published xxxxxx

\begin{abstract}
The generation of acoustic waves in structured nanobeams containing optomechanic cavities is a topic of current interest. In this paper, we study theoretically the efficient conversion of surface acoustic waves (SAW) generated on a substrate by an RF electric signal into the acoustic waves of a nanobeam. First, we demonstrate the possibility of converting the SAW generated on a silicon substrate with a thin piezoelectric AlN layer into the guided mechanical modes of a straight nanobeam with a maximum efficiency of $-22.6 \mathrm{~dB}$. This can be achieved by using a set of Inter-Digitated Transducers (IDT) with radial fingers, which provide SAW focusing towards the nanobeam entrance. We analyze the nature of the modes created inside the nanobeam and the conversion efficiency of the SAW into the different modes. Then, we study the interaction of these waves with a one-dimensional (1D) phononic crystal nanobeam containing a cavity. The latter is constituted by an array of holes and stubs and has been proven in our recent works to be very efficient for optomechanic applications. We calculate the transmission properties of the cavity modes and discuss the efficiency of generating the cavity modes from the initial SAW.
\end{abstract}

Keywords: nanobeam, acoustic waves, phononic cavity modes, IDT, SAW, phoxonic crystals

\section{Introduction}

A great deal of attention has been devoted during the last decade to the study of cavity opto-mechanics (OM) and to their potential applications from sensing to quantum information ${ }^{1,2,3,4}$. Additionally, there is a recent interest towards the realization of phononic circuits based on the coupling between several OM cavities through phononic waveguides ${ }^{1}$. Indeed, the coherent 
generation and detection of phonons by the OM cavities will open the route to the possibility of carrying and processing the information by means of phonons. In parallel, the excitations of phonons in an OM cavity by means of a Surface Acoustic Wave (SAW) generated on a piezoelectric substrate in front of the photonic/phononic crystal containing the cavity has been proposed and investigated during the last few years ${ }^{5,6,7,8}$.

The design of such OM structures provides the existence of forbidden zones (band gaps) for propagating modes in which strongly localized optical and mechanical cavity modes appears by introducing defects in a perfect periodic structure. This gives the opportunity for confinement, guiding or filtering of elastic and optical waves. The combination of phononic and photonic properties into a single platform provides a new tool for controlling of light and sound simultaneously. Currently, such artificial materials are termed phoXonic crystals ${ }^{9,10}$ or opto-mechanical crystals ${ }^{11,10}$. Several materials and nanostructures have been proposed in order to obtain simultaneous photonic and phononic band gaps. The dominant material platform is often a silicon slab or nanobeam drilled with periodic arrays of sub-micrometer holes ${ }^{8,9}$ or snow-flakes inclusions ${ }^{12}$. Following recent advances in nanotechnology fabrication, such nanostructures are currently and precisely manufactured using silicon-oninsulator (SOI) technology, 4,11 .

However, the OM coupling is currently studied mainly by additional external optical fiber ${ }^{4,11}$ placed near the OM quasiperiodic structure. The main disadvantage of this method is relatively low phonon population induced by light. Alternative way to induce large phonon population is the use of phonon pump by transformation of RF signal into surface acoustic waves (SAW). To our knowledge, the excitation of the phononic cavity mode by a SAW produced by RF pulses was first demonstrated on a two-dimensional quasi-periodic array of holes in a GaAs membrane in the $\mathrm{GHz}$ range ${ }^{6}$. In addition, the manipulation of localized mechanical modes using the radiofrequency field through a piezoelectric effect in a GaAs-based hollow-cavity optomechanical system with a straight nanobeam system supporting co-localized $1550 \mathrm{~nm}$ photons and $2.4 \mathrm{GHz}$ phonons was experimentally demonstrated for an IDT system with parallel fingers ${ }^{5,8}$ and with curved fingers ${ }^{7}$.

By focusing on the phonon propagation aspect, the objective of this work is to study the efficiency of inserting a SAW generated on a silicon substrate covered by a thin piezoelectric AIN layer and a set of inter-digitated transducers (IDT) to a nanobeam containing an OM cavity and then the excitation of the localized mechanical modes inside the cavity. The OM cavity considered in our work was proposed some years ago by some of the authors ${ }^{13,14,15}$. It is constituted by an array of holes and side stubs which are respectively favorable for the opening of a photonic and a phononic band gap ${ }^{13,14}$

The efficiency of the SAW coupling is first tested in the case of insertion into a silicon nanoplate (section 2) before being considered for a straight silicon nanobeam (section 3). In the latter case, curved IDTs are considered in order to focus a significant part of the SAW energy towards the entrance of the nanobeam, and the enhancement of the insertion is tested by means of a taper added at the nanobeam input. In section 4, we discuss the interaction of the acoustic waves inserted into the straight nanobeam with the phononic structuration mentioned above, including a cavity inside the phononic crystal. The conclusions are presented in section 5 .

\section{Electromechanical coupling in the system of IDT on piezoelectric layer deposited on Silicon membrane}

To transmit or detect mechanical waves to a Si-membrane by using SAW excited in a piezoelectric material using IDT's, we consider a multilayer structure, for which the section is schematically shown in Fig. 1. This figure shows a circuit of two opposing IDTs connected via a Si membrane. Moreover, the central region of a Si-membrane can be structured in order to obtain a straight or structured nanobeam (NB). The multilayered structure is composed of an array of Al IDT's deposited on an AlN piezoelectric material, a thin $\mathrm{Si}$ membrane, a $\mathrm{SiO}_{2}$ layer and finally the $\mathrm{Si}$ substrate, ended with a prefect matched layer (PML) for the absorption of radiative waves in numerical simulations performed by the Finite Element method. 


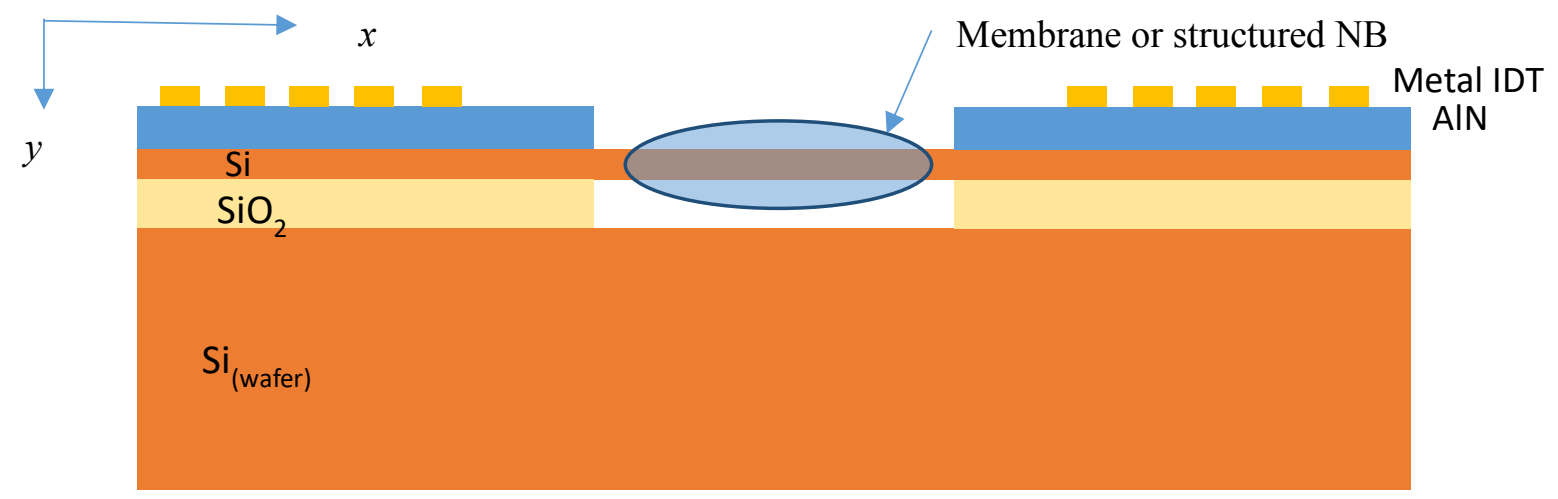

Fig. 1 : Schematic representation of a prototype device for the phonon generation and detection by electrical means using IDT on a piezoelectric AIN film deposited on the Si membrane. The surface acoustic wave generated by the IDT propagates through the Si-membrane (or through the nanobeam, where optomechanical effects can occur) and can be detected by another IDT system.

The first objective is related with the generation of surface acoustic waves on the multilayer structure with a relatively good confinement in the Si membrane which should carry the wave into the suspended membrane. For this purpose, we consider a periodic structure with the unit cell shown in Fig. 2a and present the corresponding dispersion curves in Fig. 2b for a few values of the lattice parameter, LIDT. Periodic boundary conditions are applied on each side of the unit cell, constituting the periodic structure. The set of geometrical parameters was chosen as follows: the thickness and width of Al-electrodes: $h_{\mathrm{Al}}=100 \mathrm{~nm}$ and $w_{\mathrm{Al}}=L_{\mathrm{IDT}} / 4$; the thickness of the piezoelectric layer, $h_{\mathrm{AIN}}=0.5 \mu \mathrm{m}$; the Si-membrane thickness, $h_{\mathrm{Si}}=220 \mathrm{~nm}$; the thickness of the silicon dioxide layer, $h_{\mathrm{SiO} 2}=560 \mathrm{~nm}$ and the wafer thickness bounded by a PML layer from bottom, $h \mathrm{si}($ wafer $)=7 \mu \mathrm{m}$. The excitation of the electrodes has been done at an applied voltage of $1 \mathrm{~V}$. Al, $\mathrm{Si}$ and $\mathrm{SiO}_{2} \mathrm{materials}$ are considered as cubic materials and their elastic constants are given in Table 1. For AlN, we used the following anisotropic mechanical parameters: $c_{11}=345 \mathrm{GPa}, c_{12}=125 \mathrm{GPa}, c_{13}=120 \mathrm{GPa}, c_{33}=389 \mathrm{GPa}, c_{44}=118 \mathrm{GPa}$ and $c_{66}=110 \mathrm{GPa}$, and a mass density of $3260 \mathrm{~kg} / \mathrm{m}^{3}$. The simulation was performed by using finite-element solver in COMSOL Multiphysics v5 with a piezoelectric and structural mechanics packages.

Table 1 Elastic constants and mass density for cubic materials used in the simulation

\begin{tabular}{c|cccc}
\hline & $c_{11}, \mathrm{GPa}$ & $c_{12}, \mathrm{GPa}$ & $c_{44}, \mathrm{GPa}$ & $\rho, \mathrm{kg} / \mathrm{m}^{3}$ \\
\hline $\mathrm{Si}$ & 166 & 64 & 79.6 & 2330 \\
\hline $\mathrm{Al}$ & 111.84 & 60.08 & 25.88 & 2695 \\
\hline $\mathrm{SiO}_{2}$ & 78.3 & 15.7 & 31.3 & 2200 \\
\hline
\end{tabular}

The dispersion curves presented in Fig. 2(b) are presented only for modes strongly localized in the region of the piezoelectric material. To evaluate the degree of confinement, we used the ratio between the integrals of the square modulus of the displacement, where the integration is performed over the region near top surface with IDTs, which include the AlN- and Simembranes, and the integration across the entire system including the PML region. This makes it possible to eliminate both substrate as well as PML artificial modes. In addition, sound lines corresponding to the longitudinal sound velocities in Si and $\mathrm{SiO}_{2}$ are presented in Fig. 2(b). Since the system under consideration is placed on a silicon substrate, the SAW dispersion is situated below the sound lines for the longitudinal and transverse velocities for silicon (the sound line corresponding to the transverse sound velocity in $\mathrm{Si}$ is almost equal to the longitudinal sound velocity in $\mathrm{SiO}_{2}$, thus for the sake of clarity we did not present it in Fig. 2(b)). Moreover, at the limit of the Brillouin zone, this dispersion branch is folded into the radiative frequency region. With this, the SAW localization is preserved, as can be seen from the spatial distribution of the eigenmode displacement field (the absolute value of the displacement and the corresponding strain field), which is shown in Fig. 2(c) for the case of the folded dispersion branch at the center of the Brillouin zone, at a frequency of $2 \mathrm{GHz}$ for an IDT period of $2.2 \mu \mathrm{m}$. 
a)

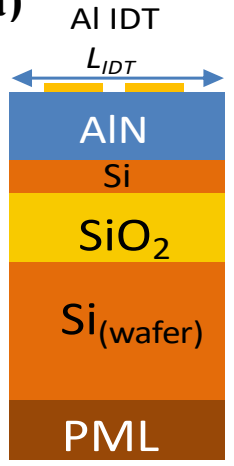

b)

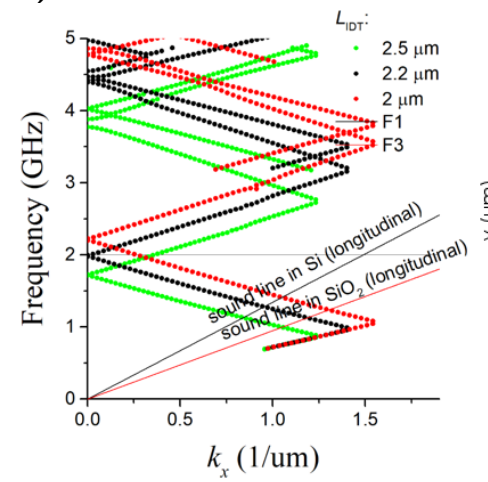

c)

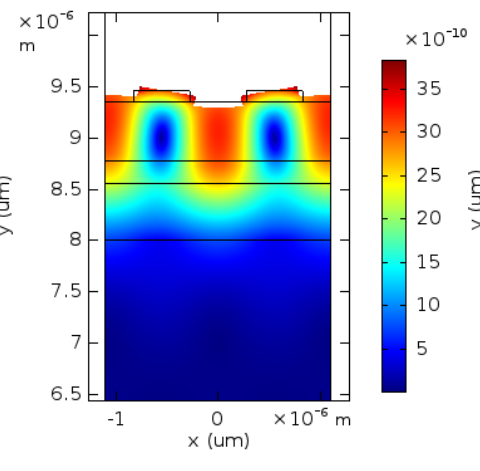

)

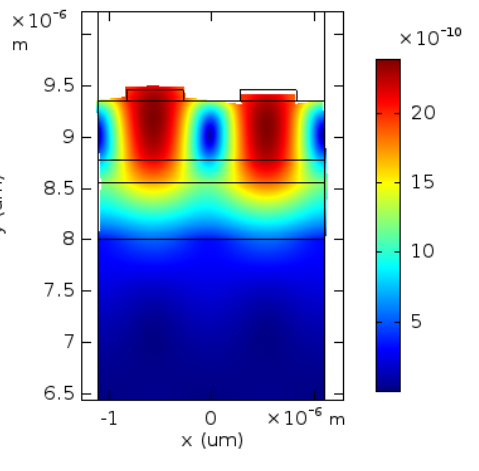

Fig. 2 : (a) Schematic presentation of the unit cell of IDT. (b) Dispersion curves corresponding to the generation of SAW for different lattice lengths: $L_{\mathrm{IDT}}=2 ; 2.2$ and $2.5 \mu \mathrm{m}$. Other geometrical parameters are $h_{\mathrm{Al}}=100 \mathrm{~nm}$ and $w_{\mathrm{Al}}=L_{\mathrm{IDT}} / 4 ; h_{\mathrm{AIN}}=$ $500 \mathrm{~nm} ; h_{\mathrm{si}}=220 \mathrm{~nm} ; h_{\mathrm{SiO} 2}=560 \mathrm{~nm} ; h_{\mathrm{Si} \text { (wafer) }}=7 \mu \mathrm{m}$. (c) Spatial distribution of the absolute value of the displacement field (in $\mathrm{m}$ ) and the corresponding strain at $2 \mathrm{GHz}$ for a lattice length of $2.2 \mu \mathrm{m}$.

We then turn to the study of the transmission of the SAW generated on the half-infinite structure into the Si nanoplate. Physically, it means that the SAW should be converted in Lamb waves. The geometrical connection is done roughly, i.e. with a continuity of the $220 \mathrm{~nm}$ silicon nanoplate while the $\mathrm{AlN}$ and $\mathrm{SiO}_{2}$ layers are stopped at the edge of the substrate (see Fig. 1). The displacement field can be seen in Fig. 3(a) from the calculation in the frequency domain at a frequency of 2 GHz. PML are added to the model from left and right sides to absorb the outgoing waves and to avoid any reflection from the boundaries of the super cell. To generate the elastic displacement, we used ten Al IDT fingers. Calculations of the mechanical flux inside the Si-nanoplate showed that the highest values of this flux could be achieved when the intersection of the SAW dispersion branch occurs in the region of zero wave vectors, which corresponds to a standing wave in the IDT region. The lattice parameter of $2.21 \mu \mathrm{m}$ yields the maximum mechanical flux in Si-nanoplate at a frequency of $2 \mathrm{GHz}$. In addition, the optimization of the thicknesses of the $\mathrm{AlN}$ and $\mathrm{SiO}_{2}$ layers give us $h_{\mathrm{AIN}}=580 \mathrm{~nm}, h_{\mathrm{SiO} 2}=560 \mathrm{~nm}$. Fig. 3(b) shows the mechanical flux inside Sinanoplate generated by electrical means over the frequency range $1-3 \mathrm{GHz}$ for a detector extended along the nanoplate section, at the right side, just before the PML. The spectrum of the mechanical flux in Si-nanoplate shows clearly the propagation of the wave inside the Si-nanoplate, and the efficient conversion of SAW into Lamb waves. The efficiency of this conversion of the RF signal into the Lamb waves (nanoplate modes) is shown in Fig. 3(c) where the maximum efficiency of about $-21 \mathrm{~dB}$ is clearly observed at a RF frequency of $2 \mathrm{GHz}$. The conversion efficiency of the electric energy to mechanical energy of Lamb waves is calculated as the ratio between the time-averaged Poynting vector integrated over the section of nanoplate and the apparent electric power consumption calculated as an integral of a product of the normal component of the current density of the anode and applied voltage over the line of the IDT anode contacts. In order to physically understand the nature of the generated waves, the spatial Fourier transform of the $x$-component of the elastic field inside the nanoplate, along the direction of propagation, is presented in Fig. 3(d) for symmetric and antisymmetric Lamb waves. As seen in Fig. 3(d), it results in the presence of two peaks at corresponding wave numbers $q=1.6 \mu \mathrm{m}^{-1}$ for symmetric and $q=5.4 \mu \mathrm{m}^{-1}$ for antisymmetric waves. These two wave vectors are in good agreement with those obtained from the dispersion curves of the Lamb modes in a $220 \mathrm{~nm}$ Si nanoplate at a frequency of $2 \mathrm{GHz}($ Fig. 3(e)). 

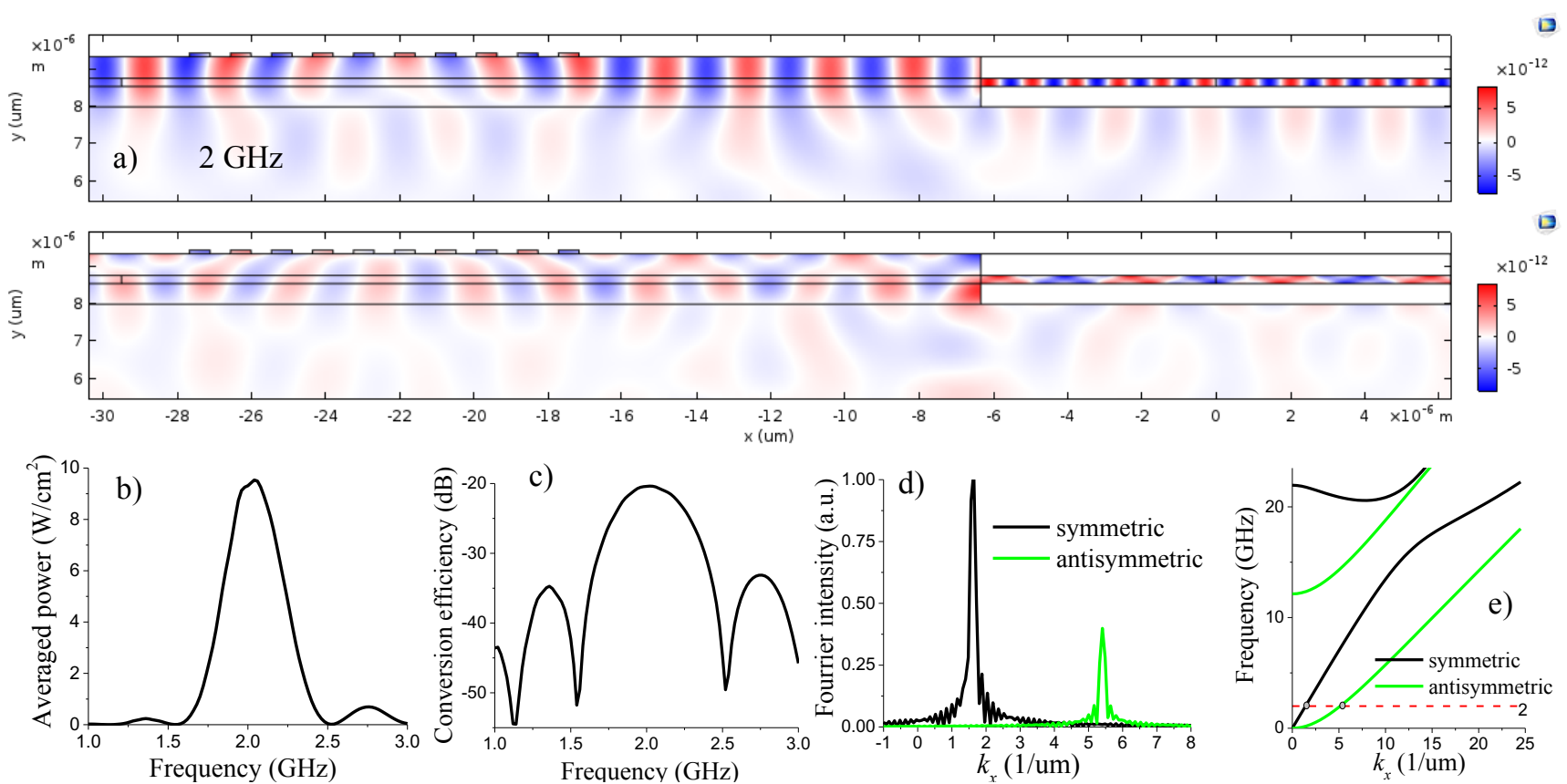

Fig. 3 : (a) Calculated real parts of the components $u_{x}$ (top) and $u_{y}$ (bottom) of the displacement field (in $\mathrm{m}$ ) at $2 \mathrm{GHz}$. (b) The power flux averaged over the nano-plate section at the right side (just before the PML). (c) Efficiency of conversion of RF signal into nano-plate modes. (d) Spatial Fourier transform for symmetric and anti-symmetric waves for a linear crosssection through a nano-plate in the direction of propagation at a frequency of $2 \mathrm{GHz}$. The resulting wave vectors coincide well with those of the Lamb modes of the $220 \mathrm{~nm}$ Si nanoplate at $2 \mathrm{GHz}$, as shown in panel (e). The other geometrical parameters are $h_{\mathrm{Al}}=100 \mathrm{~nm}$ and $w_{\mathrm{Al}}=L_{\mathrm{IDT}} / 4 ; h_{\mathrm{AlN}}=580 \mathrm{~nm} ; h_{\mathrm{Si}}=220 \mathrm{~nm} ; h_{\mathrm{SiO} 2}=560 \mathrm{~nm}$.

In conclusion of this section, we demonstrated the generation of SAW in the multilayer structure under consideration and it was shown that this SAW could be efficiently converted into the Lamb modes of a nano-plate.

\section{Efficiency of the conversion from RF electrical signal to the guided modes of a straight nanobeam}

In this section, we analyze the possibility of the effective insertion of SAW into a nanobeam (NB). To do this, one considers a system in which a Si-membrane is replaced with a straight NB or a structured NB containing a phoxonic crystal with a cavity. Thus, membrane modes are replaced by NB-modes, and we need to study the conversion of SAW to NB-modes.

While our system has in-plane confinement, we firstly analyzed the shape of the fingers that form the IDT for the conversion of SAW into the modes of NB with a rectangular shape. Fig. 4 shows the spatial distribution of the longitudinal component of the displacement field in the transition region, from the plate with the IDT system to the straight NB. Three types of IDT structure (as shown in Fig. 4) are tested, namely: (i) parallel fingers, (ii) radially curved fingers while the NB is directly connected to the straight edge of the plate and (iii) radially curved fingers while adding a taper at the connection between NB and the straight edge of the plate. The set of geometrical parameters have been chosen as: $h_{\mathrm{Al}}=100 \mathrm{~nm}$ and $w_{\mathrm{Al}}=L_{\mathrm{IDT}} / 4$; $h_{\mathrm{AIN}}=250 \mathrm{~nm} ; h_{\mathrm{Si}}=220 \mathrm{~nm} ; h_{\mathrm{SiO} 2}=1 \mu \mathrm{m} ; h_{\mathrm{Si} \text { (wafer) }}=4 \mu \mathrm{m}$, the number of fingers was chosen to be 7 and the excitation of the electrodes has been done at an applied voltage of $1 \mathrm{~V}$. The geometrical parameters of the rectangular shape NB are the width $a=500 \mathrm{~nm}$ and the height, $e=220 \mathrm{~nm}$ which coincides with the thickness of Si membrane. As we can see from Fig. 4 , the transfer of the mechanical energy of SAW into the NB is inefficient when the IDT's are straight. Indeed, the mechanical waves remain mostly localized at the edge of the Si plate due to SAW reflection from this edge. The radially curved fingers provide better opportunity for SAW conversion into NB modes due to the SAW focusing near the region where the NB is connected to the edge of the Si layer. This can be improved by using an additional taper (Fig. 4(c)) which has the ability of adiabatically pulling the right edge of the Si layer into the NB. All the next simulations were performed for this last design. The thicknesses of the AlN and $\mathrm{SiO}_{2}$ layers: $h_{\mathrm{AIN}}=250 \mathrm{~nm}$ and $h_{\mathrm{SiO} 2}=1 \mu \mathrm{m}$ were chosen from conventional fabrication issues. 

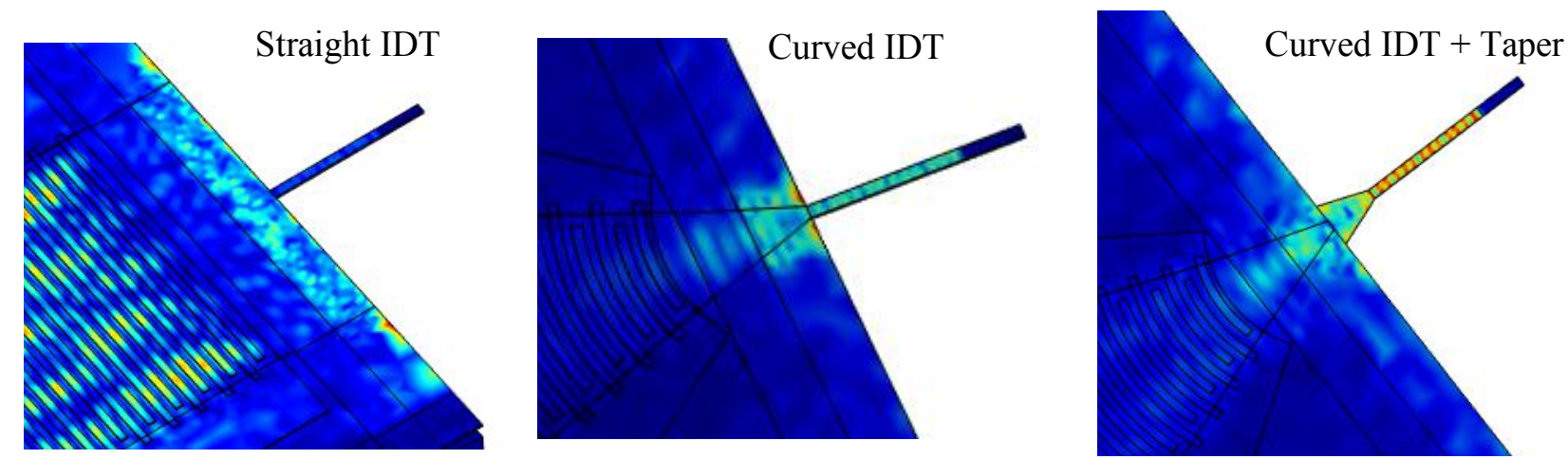

Fig. 4 : The spatial distribution of the longitudinal component of the displacement field for IDT terminated by a nanobeam. Three different IDT structure have been tested. The same scale of the displacement field is used for these three cases.

To analyze the nature of the SAW conversion to NB modes, it is necessary to consider the behavior of the dispersion curves for the straight NB with a rectangular shape of section $a \times e$, as shown in Fig. 5. One can see the occurrence of four propagative low-frequency branches with different phase velocities starting from the origin. The parity of the modes with respect to the symmetry planes $\Pi$ and $\Pi$ ' (shown in the inset of Fig. 5) are summarized in Table 2. The spatial distributions of the displacement fields for these first four low-frequency eigenmodes are presented in Table 2. Each mode is represented according to the real parts of the components $u_{x}, u_{y}$, and $u_{z}$ of the displacement field $\tilde{u}_{i}(x, y, z, t)=u_{i}(y, z) e^{-i k_{\text {mode }} x+i \omega t}$, where $k_{\text {mode }}$ is the wave vector of mode $(i)$. All four modes are orthogonal to each other that avoids the coupling between them.

Table 2 Symmetry of the first four low frequency modes of the rectangular nanobeam. The parity even (e) and odd (o) are defined with respect to the plane $\Pi$ and $\Pi$ ' respectively (see Fig. 5). Spatial distribution of the displacement field for the first four low-frequency modes at a frequency of $2 \mathrm{GHz}$.

\begin{tabular}{c|cccccc}
\hline mode & $\Pi^{\prime}$ & $\Pi$ & $\begin{array}{c}\text { Parity } \\
\text { (Symmetry) }\end{array}$ & $\begin{array}{c}\text { Spatial distribution of the displacement field } \\
\operatorname{Re}\left(u_{x}\right)\end{array}$ & $\operatorname{Re}\left(u_{y}\right)$ & $\operatorname{Re}\left(u_{z}\right)$ \\
\hline 1 & even & even & ee (S'S) & & \\
\hline 2 & odd & odd & oo (A'A) & & \\
\hline 3 & odd & even & oe (A'S) & & \\
\hline 4 & even & odd & eo (S'A) & & \\
\hline
\end{tabular}




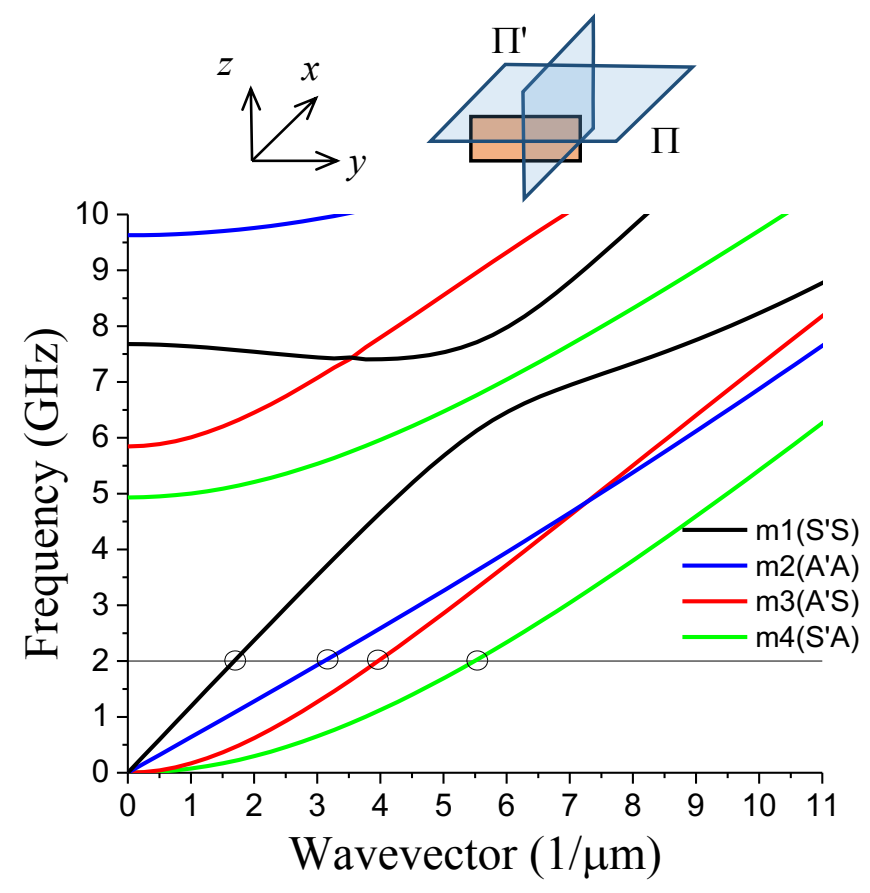

Fig. 5 : Dispersion curves of the silicon nanobeam of rectangular section $(a \times e)=(500 \mathrm{~nm} \times 220 \mathrm{~nm})$.

Using the spatial distribution of the straight NB eigenmodes, the displacement field of the wave which has reached the NB from the SAW generated by the IDT's can be written in the superposition form

$$
\mathbf{u}=\sum_{i=1}^{N} c_{i} \mathbf{u}_{i}
$$

where $\mathbf{u}_{i}$ is the displacement vector for each NB mode, $c_{i}$ is the decomposition coefficient, $N$ is the number of NB modes $(N=$ 4 for low frequency region). Since the eigenmodes are orthogonal, the decomposition coefficients can be determined from Eq. (1) by integration over NB section, $S_{N B}$, in the following form

$$
c_{i}=\frac{\iint_{S_{N B}} \mathbf{u}_{i}^{+} \mathbf{u} d S}{\iint_{S_{N B}} \mathbf{u}_{i}^{+} \mathbf{u}_{i} d S} .
$$

To analyze the conversion efficiency of the electric energy to mechanical energy of NB modes we use the ratio between the time-averaged Poynting vector integrated over the section of NB and the apparent electric power consumption in the following form

$$
\mathrm{CE}=\frac{\iint_{S_{N B} P_{\text {avx }} d S}}{\iint_{S_{\text {anode }}\left|J_{n}^{*} V\right| d S},}
$$

where $P_{a v x}$ is the time-averaged $x$-component of the Poynting vector that is normal to the NB section with the area, $S_{N B}, J_{n}$ is the normal component of the current density of the anode (or ground), $V$ is the applied voltage (assumed to be 1 volt), $S_{\text {anode }}$ is the surface of the IDT anode contacts.

The power of guided NB-modes and the efficiency of conversion of electrical energy into mechanical energy of NB-modes using Eq. (3) are shown in Fig. 6 for all NB modes and separately for each mode. The conversion efficiency is calculated for the time-averaged $x$-component of the Poynting vector in the T-section of NB (see the inset in Fig. 6(a)) taking into account the decomposition coefficients (Eq. (2)) of the displacement field. As can be seen from Fig. 6(b), the conversion efficiency from electrical energy to mechanical energy for all NB modes reaches a maximum of $-22.6 \mathrm{~dB}$ at a frequency of $2 \mathrm{GHz}$. Only modes with in-plane symmetry can be effectively excited in the system under consideration. 

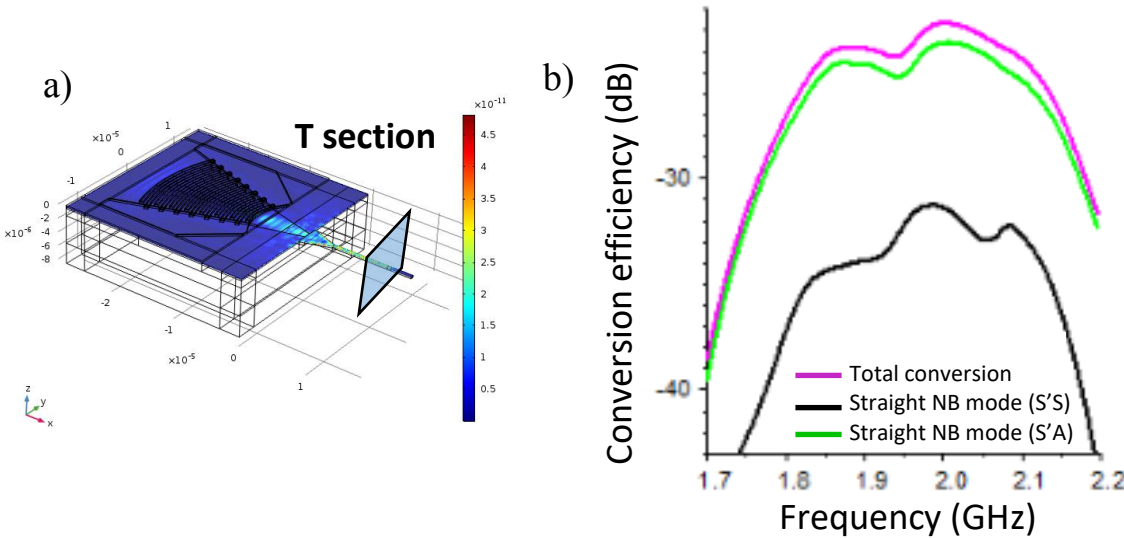

Fig. 6 : (a) Spectrum of the $x$-component of the Poynting vector averaged over T plane (the end of straight NB before PML region in the full piezoelectric simulation). The inset presents the spatial distribution of the displacement field in Silicon at $2 \mathrm{GHz}$. (b) Efficiency of the conversion from the electric signal to mechanical modes of a straight NB.

\section{Excitation of cavity modes in a structured nanobeam}

In this section, we assume that in continuation of the straight NB, there is a structured NB constituting a 1D phononic crystal, possibly containing a cavity. This structured NB consists of a 1D quasi-periodic structure of holes and stubs with a cavity defect breaking the periodicity at the center of the quasi-periodic structure ${ }^{14,14}$ (see Fig. 7). The defect is designed by adiabatic variation of the width of perfect cells as well as the variation of hole diameters and stub widths. This adiabatic variation of cell parameters leads to high Q-factors for optical and for mechanical cavity modes. This structure has been proven to exhibit efficient $\mathrm{OM}$ behaviors with high coupling rate for $\mathrm{GHz}$ phonons. The shape and geometrical parameters of the structure in Fig. 7 were taken from ${ }^{15}$. The band diagram of the periodic system consisting of the perfect cell is presented in Fig. 7(a) where we limit ourselves only to the two NB modes which are symmetrical (referred to as S') with respect to the $x \mathrm{O} z$-plane ( $\Pi$ ' plane in Fig. 5). These two types of modes are respectively symmetrical and anti-symmetrical with respect to the $\Pi$ plane in Fig. $\mathbf{5}$ and for this reason we refer to them as S'S and S'A. One can observe in Fig. 7 (a) the existence of a full band gap in the range of 1.8-2.0 GHz for these two types of modes, since there is a band gap of 1.8-2.2 GHz for the S'S mode and a band gap of 1.6$2 \mathrm{GHz}$ for the S'A mode.

Fig. 7(b) presents the efficiency of the conversion from the electric energy applied to IDT to the energy of each NB modes with the symmetry S' in the structured NB. We also show the conversion efficiency to the modes of the straight NB by dashed lines, as well as the total conversion from Fig. 6. The simulation was carried out in two steps. The first step includes the generation of SAW until their insertion into the straight NB (Fig. 6). The second step considers the scattering of the modes generated in the straight NB and discussed in the previous section with the structured 1D phononic crystal and its cavity. In Fig. 7(b), we can observe the formation of the band gap for the NB modes with the $x \mathrm{O} z$-plane symmetry with extremely sharp peaks corresponding to the cavity modes of the structured NB inside the bandgap. The spatial distribution of the absolute value of the dimensionless displacement field are presented in Fig. 7(c) for two S'S cavity modes near 2 GHz. 

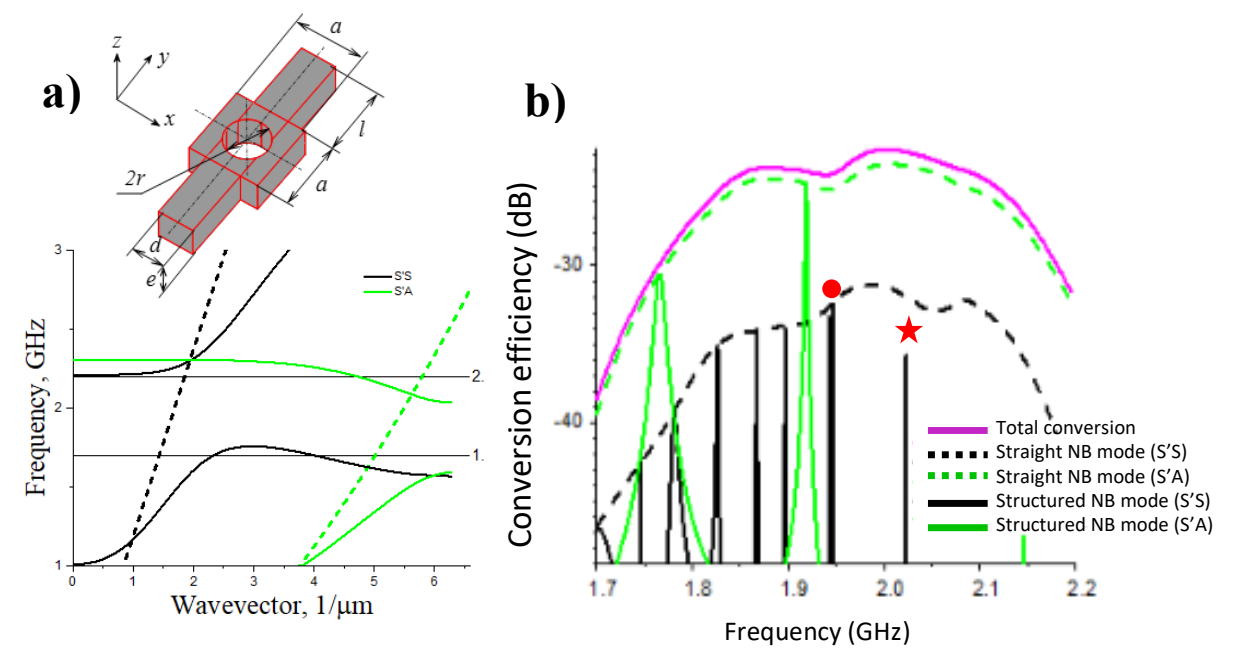

c)

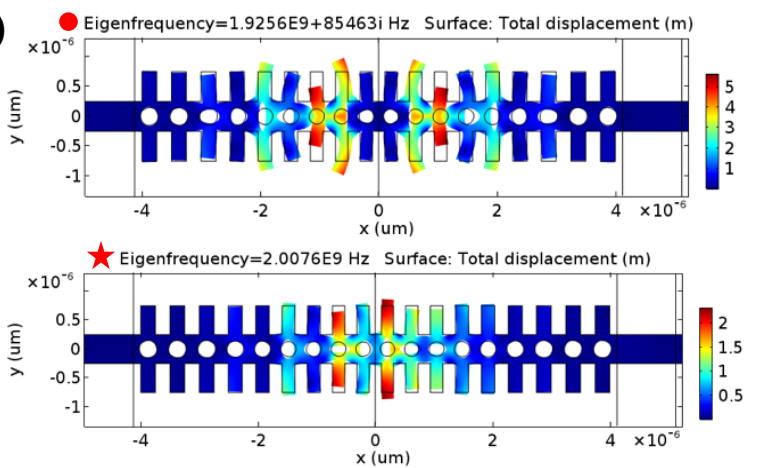

Fig. 7 : (a) Band diagram for periodically structured NB with the unit cell presented in the inset. Parameters of structured NB: $a=500 \mathrm{~nm}$ is the lattice parameter of a quasi-periodic structuration of NB, $e=0.44 a$ is the thickness of the NB, $r=0.3 a$ is the radius of the hole, and $d=0.5 a$ and $l=a$ are the width and the length of one rectangular stub, respectively. (b) Spectrum of the SAW conversion efficiency for each NB modes and the SAW conversion into all NB modes with S' symmetry. (c) Displacement field distribution for the S'S cavity mode in the vicinity of $2 \mathrm{GHz}$.

\section{Discussions and conclusions}

This paper represents a progress in the chip based electro-mechanical devices for further applications in the area of sensing, metrology, and quantum information science. We have shown the possibility of an efficient conversion of an electrical RF signal into NB mechanical modes. We found that the best conversion could be achieved with radially curved fingers of IDT, which perform the SAW focusing, and then a smooth transition of the straight edge of the silicon film of the multilayer substrate into a straight silicon NB with a rectangular section. The resulting maximum efficiency reaches a value of $-22.6 \mathrm{~dB}$. This value is reasonable and is consistent with that for the $\mathrm{ZnO}$-based piezoelectric system, where the efficiency of conversion of an electrical RF signal into SAW of more than $1 \%$ was shown ${ }^{16}$. Notice, that in our case, in contrast to this $\mathrm{ZnO}$-based piezoelectric system, there is additional transformations of SAW into silicon membrane modes and next to guided modes of a straight NB, therefore the obtained value is very promising. In addition, there are ways to increase the conversion efficiency. Namely, it is possible to reduce the backward propagation of SAW (in the opposite direction from the NB) due to the use of a reflector, for example by using a Bragg mirror. In this case, the efficiency can be almost doubled. Moreover, the number of fingers in the IDT system can be increased or the complex finger configuration can be used (for example, doubled fingers).

It was shown that in the IDT system under consideration, only two low-frequency NB modes (of which one is longitudinal) are excited, which are symmetrical with respect to the $x \mathrm{O} z$-plane of the NB. This is not a surprise since this IDT system is almost symmetrical with respect to this plane. The maximum conversion efficiency for them are $-23.6 \mathrm{~dB}$ for the S'S mode and $-31.3 \mathrm{~dB}$ for the S'A mode. In addition, the possibility of the excitation of cavity modes in the case of structured NB was numerically demonstrated in this paper. This gives the right to assert that this configuration can be used for efficient pump of cavity phonons by the electrical RF signal in the case of a more complicated configuration including an optomechanical coupling with one or more additional NBs. In addition, the IDT system under consideration can be used not only for phonon 
pumping, but also for detecting the NB cavity phonons excited due to optomechanical coupling with other external NBs (which can be on-chip implemented). For detection efficiency, i.e. NB to RF conversion, our simulations give us the value of the same order as for RF to NB conversion. For example, the maximum value of the conversion from the longitudinal NB mode to the RF electrical signal is $-34.5 \mathrm{~dB}$, whereas the corresponding value for the conversion from the RF electrical signal to the longitudinal NB mode is $-31.3 \mathrm{~dB}$ as mentioned above. Thus, the total efficiency of RF to RF conversion in the case of two IDT systems under consideration (one for SAW excitation on one side of the NB, and the other for detecting the RF signal on the opposite side of the NB) can be estimated as double the efficiency of the RF to NB modes conversion only.

In addition, it was found that the maximum displacement reaches a value of $30 \mathrm{pm}$ in the area of straight NB in the system under consideration when an RF signal of $1 \mathrm{~V}$ is applied at a frequency of $2 \mathrm{GHz}$. Then, the transmission simulation showed an increase in the total displacement up to 100 times in the case of a structured NB when the cavity mode is excited. This means that the maximum deformation may be about $3 \mathrm{~nm}$ in the case of RF excitation in the system under consideration with a structured NB.

\section{Acknowledgements}

This work was supported by the European Commission H2020 project PHENOMEN, under Grant Agreement No. 713450

\section{References}

1 Kejie Fang, Matthew H. Matheny, Xingsheng Luan and Oskar Painter, Nature photonics 10, 489 (2016).

2 Aspelmeyer M., Kippenberg T.J. and Marquardt F 2014 Rev. Mod. Phys. 861391

3 Gavartin E., Braive R., Sagnes I., Arcizet O., Beveratos A., Kippenberg T. J., and Robert-Philip I. 2011 Phys. Rev. Lett. 106203902

4 Maire J., Arregui G., Capuj N.E., Colombano M.F., Griol A., Martinez A., Sotomayor-Torres C.M., Navarro-Urrios D. 2018 APL Photonics 3126102

5 Balram K.C., Davanco M., Dong Song J., Srinivasan K. 2016 Nature Photonics 10346

6 Fuhrmann D.A., Thon S.M., Kim H., Bouwmeester D., Petroff P.M., Wixforth A. and Krenner J.H. 2011 Nature Photonics 5605

7 Vainsencher A., Satzinger K. J., Peairs G. A., and Cleland A. N. 2016 Appl. Phys. Let. 109033107

8 Balram K.C., Davanço M.I., Ilic B.R., Kyhm J.-H., Dong Song J., and Srinivasan K. 2017 Phys. Rev. appl. 7024008

9 Maldovan M. 2013 Nature $\mathbf{5 0 3} 209$

10 Pennec, Y., Rouhani, B. D., El Boudouti, E. H., Li, C., El Hassouani, Y., Vasseur, J. O., Papanikolaou N., Benchabane S., Laude V.and Martinez, A. 2010 Optics express 1814301

11 Eichenfield M., Chan J., Camacho R.M., Vahala K.J., and Painter O. 2009 Nature 46278

12 Safavi-Naeini A. H., Hill J. T., Meenehan S., Chan J., Gröblacher S. and Painter O. 2014 Physical review letters 112 153603.

13 Pennec Y., Djafari Rouhani B., Li C., Escalante J. M., Martinez A., Benchabane S., Laude V. and Papanikolaou N. 2011 AIP Advances 1041901

14 Oudich M., El-Jallal S., Pennec Y., Djafari-Rouhani B., Gomis-Bresco J., Navarro-Urrios D., Sotomayor Torres C.M., Martinez A. and Makhoute A. 2014 Phys. Rev. B 89245122

15 Gomis-Bresco J., Navarro-Urrios D., Oudich M., El-Jallal S., Griol A., Puerto D., Chavez E., Pennec Y., Djafari-Rouhani B., Alzina F., Martínez A. and Sotomayor Torres C.M. 2014 Nature Communications 54452

16 Hashimoto K. "Surface Acoustic Wave Devices in Telecommunications: Modelling and Simulation", p.186. 